\title{
ARTICLE
}

\section{Latent variable analysis of negative affect and its contributions to neural responses during shock anticipation}

\author{
Namik Kirlic (iD) ${ }^{1}$, Robin L. Aupperle ${ }^{1,2}$, Jamie L. Rhudy ${ }^{3}$, Masaya Misaki ${ }^{1}$, Rayus Kuplicki ${ }^{1}$, Anne Sutton ${ }^{1}$ and Ruben P. Alvarez ${ }^{1}$
}

Negative affect is considered an important factor in the etiology of depression and anxiety, and is highly related to pain. However, negative affect is not a unitary construct. To identify specific targets for treatment development, we aimed to derive latent variables of negative affect and test their unique contributions to affective processing during anticipation of unpredictable, painful shock. Eighty-three subjects (43 with depression and anxiety spectrum disorders and 40 healthy controls) completed self-report measures of negative valence and underwent neuroimaging while exploring computer-simulated contexts with and without the threat of a painful, but tolerable, shock. Principal component analysis (PCA) extracted distinct components of general negative affect (GNA) and pain-related negative affect (PNA). While elevated GNA and PNA were both indicative of depression and anxiety disorders, greater PNA was more strongly related to task-specific anxious reactivity during shock anticipation. GNA was associated with increased precuneus and middle frontal gyrus activity, whereas PNA was related to increased bilateral anterior insula activity. Anterior insula activity mediated the relationship between PNA and task-specific anxious reactivity. In conclusion, GNA and PNA have distinct neural signatures and uniquely contribute to anxious anticipation. PNA, via insula activity, may relate to arousal in ways that could contribute to affective dysregulation, and thus may be an important treatment target.

Neuropsychopharmacology (2019) 44:695-702; https://doi.org/10.1038/s41386-018-0187-5

\section{INTRODUCTION}

Depression and anxiety are some of the most common forms of mental illness [1] and the leading causes of disability worldwide [2]. Highly comorbid, they are believed to stem from high negative affect, a nonspecific vulnerability to experience chronic generalized distress, heightened anticipation of future catastrophic events, and subsequent maladaptive coping, such as excessive withdrawal from the environment [3]. Pain, an unpleasant sensory and emotional experience associated with actual or potential tissue damage [4], commonly co-occurs with negative affect $[5,6]$. In fact, research shows increased rates of comorbidity between chronic pain and depression and anxiety [7-11]. Psychological mechanisms that are relevant to the experience of pain and pain disorders are characterized by anxiety sensitivity (e.g., mental, physical, and social concerns) and illness/injury sensitivity (e.g., pain catastrophizing and fear of pain) $[12,13]$. Moreover, these processes not only contribute to pain conditions [14], but are also similarly disrupted in affective disorders in the absence of pain $[15,16]$. Therefore, although a cyclical relationship between pain and psychopathology is likely, there may be a specific contribution of pain-related emotional processes (e.g., physical concerns, pain catastrophizing, fear of pain, avoidance of physical discomfort, or pain) to the etiology of affective disorders, such as depression and anxiety.

Pain-related processes indeed appear to be a unique component of negative affect that occur in the absence of psychopathology and chronic pain. In healthy subjects, two separate principal component analyses (PCA) on a number of negative valence measures revealed separable negative affect components, including general distress, fear of pain from injury/insult, and cognitive intrusion of pain $[17,18]$. Delineating how these separable constructs might contribute to the anticipation of painful events across clinical populations, as well as their neural, physiological, and behavioral correlates, would be important for elucidating not only multi-level mechanisms involved in the processing of negative affect, but also their contribution to affective dysregulation.

Neural mechanisms relevant to pain experiences and the anticipation of pain specifically, have been explored in previous neuroimaging studies. This research implicates a network of brain regions including the anterior insula, ventromedial prefrontal cortex (vmPFC), orbitofrontal cortex (OFC), anterior midcingulate cortex (aMCC), amygdala, bed nucleus of the stria terminalis (BNST), ventral striatum (VS), hypothalamus, and hippocampus $[19,20]$. Affective disorders are characterized by alterations in this network and theorized to relate to increased threat attention and hypervigilance (amygdala, OFC, VS), inflated estimate of threat cost and probability (dmPFC, aMCC, anterior insula, and VS), heightened reactivity (amygdala, BNST, hypothalamus, and midbrain), increased behavioral and cognitive avoidance (OFC, dIPFC, striatum, aMCC, and anterior insula), and deficient safety learning (vmPFC and hippocampus).

In this study, we set out to understand the unique effect of latent negative affect variables on anxious reactivity (anxiety, skin conductance responses [SCRs], and exploratory behavior), and brain responses during anticipation of unpredictable painful shock. Although we report group differences where appropriate (healthy controls vs. individuals with depressive and anxiety

\footnotetext{
${ }^{1}$ Laureate Institute for Brain Research, Tulsa, OK, USA; ${ }^{2}$ School of Community Medicine, University of Tulsa, Tulsa, OK, USA and ${ }^{3}$ Department of Psychology, University of Tulsa, Tulsa, OK, USA

Correspondence: Namik Kirlic (nkirlic@laureateinstitute.org)
}

Received: 27 April 2018 Revised: 11 July 2018 Accepted: 14 August 2018

Published online: 22 August 2018 
spectrum disorders), we referenced the Research Domain Criteria framework and focused on a dimensional evaluation and interpretation of relevant constructs and findings. We reasoned that this would allow for further elucidation of context-relevant psychological constructs and their relationship with task-related responses, as well as have greater transdiagnostic utility going forward. Thus, healthy subjects and subjects with depressive and anxiety disorders completed a range of negative valence measures and underwent neuroimaging while completing a task designed to elicit sustained anticipation of shock. We hypothesized that a PCA would produce separable components related to general and pain-related negative affect (PNA) processes, and that each would evidence distinct neural signatures during shock anticipation. We predicted that both general and PNA would elicit increased anxious reactivity. However, we predicted that general negative affect (GNA) would relate to increased amygdala/BNST activation, while PNA would relate to increased anterior insula activity.

\section{METHODS}

\section{Participants}

Eighty-three volunteers, ages 18-50, participated in the study, including 43 with depression and anxiety spectrum disorders and 40 healthy control participants (Table 1). Diagnosis was confirmed using the DSM-IV-TR criteria with the Structured Clinical Interview for DSM-IV and an unstructured interview with a psychiatrist.

\section{Measures}

Subjects completed the Inventory of Depression \& Anxiety Symptoms, Expanded Version (IDAS-II), State Trait Anxiety Inventory (STAl), Anxiety Sensitivity Index-3 (ASI-3), Anxiety Control Questionnaire-Revised version (ACQ-R), Pain Anxiety Symptoms Scale-20 (PASS-20), and Pain Catastrophizing Scale (PCS). Measures are described in the Supplemental Information (SI).

\section{The paradigm}

The task was conducted as previously described (Fig. 1 and SI [21]). Participants were asked to virtually explore two computersimulated rooms and informed that they would be later asked

\begin{tabular}{|c|c|c|c|c|c|c|}
\hline \multirow{3}{*}{$\begin{array}{l}\text { Characteristic } \\
\text { Female }\end{array}$} & \multirow{2}{*}{\multicolumn{2}{|c|}{$\frac{\mathrm{HC}(n=40)}{50 \%}$}} & \multirow{2}{*}{\multicolumn{2}{|c|}{$\frac{\mathrm{PT}(n=43)}{65 \%}$}} & \multirow{3}{*}{$\begin{array}{l}x^{2} \\
1.37 \\
t_{81}\end{array}$} & \multirow{3}{*}{$\begin{array}{l}p \\
.24 \\
p\end{array}$} \\
\hline & & & & & & \\
\hline & Mean & SD & Mean & SD & & \\
\hline Age (years) & 30.95 & 10.15 & 32.09 & 10.37 & -.51 & .61 \\
\hline Body mass index $\left(\mathrm{kg} / \mathrm{m}^{2}\right)$ & 26.00 & 4.45 & 26.86 & 4.22 & -.90 & .37 \\
\hline US stimulus intensity (mA) & 17.55 & 7.47 & 16.38 & 7.36 & .72 & .50 \\
\hline US pain intensity (0-100 scale) & 48.84 & 14.67 & 47.31 & 14.62 & .47 & .60 \\
\hline Psychiatric diagnosis & $\mathrm{N}$ & & $\mathrm{N}$ & & & \\
\hline MDD & 0 & & 15 & & & \\
\hline PD & 0 & & 6 & & & \\
\hline MDD/PD & 0 & & 9 & & & \\
\hline GAD & 0 & & 3 & & & \\
\hline MDD/PTSD & 0 & & 6 & & & \\
\hline PTSD & 0 & & 3 & & & \\
\hline SAD & 0 & & 1 & & & \\
\hline
\end{tabular}

$H C$ healthy controls, GAD generalized anxiety disorder, MDD major depressive disorder, $P D$ panic disorder, $P T$ patients with depressiveanxiety spectrum disorders, PTSD posttraumatic stress disorder, $S A D$ social anxiety disorder, US unconditioned stimulus what they learned about each room. Instructions stated that when in the purple (or peach) room, participants could receive a shock on the ankle at any time (threat), but when in the peach (or purple) room, a shock would never be delivered (safe).

During each of the four fMRI scans, five threat and five safe contexts were semi-randomly presented for a duration of $18 \mathrm{~s}$. One to two unpredictable electric shocks were delivered per scan during threat contexts (five in total; ranging $3-16 \mathrm{~s}$ post context onset; mean onset $=9.6 \mathrm{~s}$ ). The order of scan presentation was counterbalanced across participants. Participants rated the intensity of the shock following threat contexts that included shocks. Post the scan, participants retrospectively rated how fearful they were on average in each context. Subjective anxiety and skin conductance responses were indexed by subtracting values in the safe from the threat context. Exploratory behavior was indexed as the average time spent virtually moving in threat relative to safe contexts.

Electric stimuli and skin conductance assessment

Shocks were delivered to the left ankle over the retromalleolar pathway of the sural nerve, $2 \mathrm{~cm}$ posterior to the malleolus [22]. Task stimulus intensity was set to achieve a level of stimulation necessary to reliably elicit a nociceptive flexion reflex and the subject's rating of stimulus as moderately painful. SCRs were recorded over the medial side of the right foot over the abductor hallucis muscle [23]. Data analysis was performed using the general linear convolution model-based analysis of waveforms as implemented in SCRalyze software to estimate the mean response amplitude for threat and safe contexts [24].

fMRI data preprocessing and subject-level analyses

Functional image preprocessing and analysis were performed using AFNI (http://afni.nimh.nih.gov/afni), and spatial alignment of functional images was performed using advanced normalization tools (http://stnava.github.io/ANTs) to optimize the spatial alignment of functional data to the TT_N27 T1-weighted template (see $\mathrm{SI}$ ). Single subject-level analyses were conducted using AFNI's 3dREMLfit. Task contexts were modeled as the sum of piecewise linear B-spline basis (tent) functions. Fifteen functions covering $30 \mathrm{~s}$ were used to account for the full-context duration (0-18 s) and subsequent BOLD response recovery. For the threat vs. safe context contrast, voxel-wise analysis included only regressors for the 10 time points spanning each context. Responses to threat context included only the trials in which shock was not delivered.

Principal component analysis (PCA)

Questionnaires were analyzed using a PCA in SPSS with an orthogonal (varimax) rotation in order to determine whether a smaller number of core components underlie the larger number of psychological constructs related to negative affect [25]. Highly correlated variables, largely independent of other subsets, are combined into components. Parallel analysis [26] determined the number of components retained. This method first randomly generates a dataset with the same number of cases and variables. PCA is then repeatedly performed on this dataset averaging eigenvalues across analyses. Components whose eigenvalues exceed the average eigenvalue from the randomly generated dataset are retained. A regression approach was used to generate factor scores. Only component loadings $>.80$ were interpreted [27].

Group-level analyses

Whole-brain, voxel-wise analyses using AFNI's 3dMEMA program examined the differences in threat versus safe contexts, covarying for GNA, PNA, and subject age. The results were corrected for multiple comparisons using Monte Carlo simulations. Significance criterion for whole-brain analysis was set at a corrected rate of $p<.005$ (cluster size $\geq 6$ voxels) and thresholded per-voxel at 
a

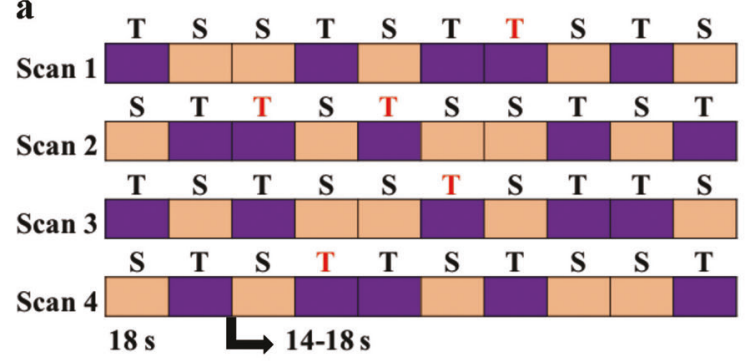

c

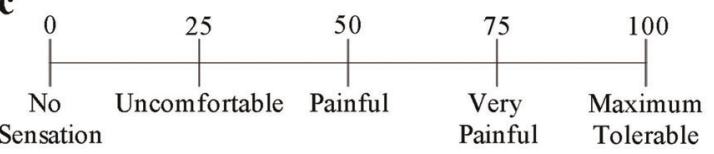

b
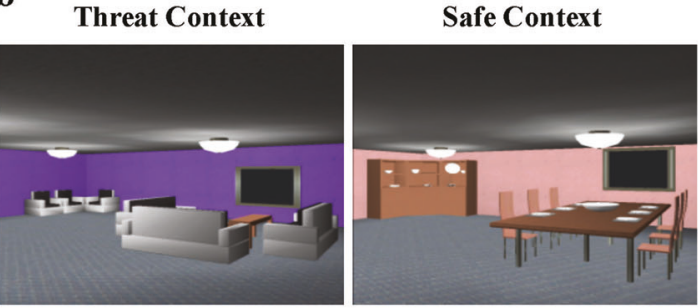

d

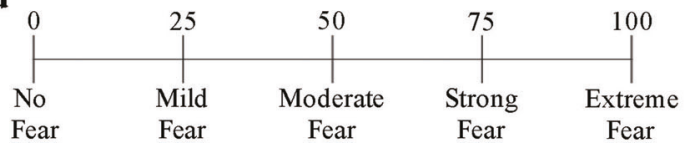

Fig. 1 Anticipation of unpredictable, painful shock task [40]. a During the task, subjects explored two contexts, one in which there was a threat $(T)$ of receiving a painful electric shock stimulation at any time, and one in which they were safe (S) from receiving any shock. The acronyms colored in red denote contextual epochs in which unsignaled shocks were administered. b Static pictures of the computersimulated rooms that served as threat and safe contexts. c Following each threat context in which an electric shock was administered, subjects rated the intensity of the shock received. d Following each scan, subjects also retrospectively rated how fearful they were in the threat and safe contexts using a 0-100 scale

$p<.00001$ for a meaningful separation of clusters (3dClustSim). Significance criterion for detecting activation for GNA and PNA factors in the whole-brain analysis was set at a corrected rate of $p<.005$ (cluster size $\geq 48$ voxels) and thresholded per-voxel at $p<.01$. Average percent signal change (PSC) was extracted per individual from significant clusters of activation identified in the whole-brain analysis. Although we focus on the relationship between negative affect components and task responses in the whole sample, we examined the effect of group differences on these results (i.e., healthy controls vs. depressed and anxious patients) and report them in the supplement.

\section{Mediation analyses}

Given the pivotal role of dorsal anterior insula (dAl) in anticipatory anxiety, simple mediation analyses tested whether dAl activity mediated the relationship between PNA and anxious reactivity. We used the ordinary least-squares path approach in the SPSS version of PROCESS [28] with a bias-corrected 95\% bootstrap confidence interval for indirect effects based on 10,000 bootstrap samples. PSC for the threat vs. safe contrast was extracted from the left and right dAI ROIs rather than from the clusters identified in the whole-brain analysis. ROls were defined a priori using prerendered stereotaxic masks available in AFNI (i.e., Talairach-Tournoux N27 atlas (1988) [29]) and adjusted to include the dorsal anterior regions (Figure S1). Given notable group differences in PNA, anxiety, and bilateral dAl, we statistically controlled for group effects.

\section{RESULTS}

Demographic and anxious reactivity data

Participant characteristics data (Table 1) indicated no significant differences between the groups in age, BMI, stimulus intensity, or subjective pain. Subjective anxiety and SCRs were greater during threat than safe contexts [ANXIETY: Threat: $M(S D)=32.91(21.33)$, Safe: $M(S D)=1.78(3.78), t_{(82)}=11.80, p<.001$; SCRs: Threat: $M(S D)$ $=.014(.02)$; Safe: $\left.M(S D)=.0003(.01), t_{(82)}=9.29, p<.001\right]$. On an average, virtually moving did not significantly differ for Threat $[M$ $(S D)=4.14(.23)]$ and Safe $[M(S D)=4.29(.24)]$ contexts $\left[t_{(82)}=4.35\right.$, $p=.18]$.

\section{PCA}

Descriptive statistics for the measure subscales are shown in Table S1. Table S2 reports correlations between the subscales revealing that they are well correlated and suitable for PCA. A twocomponent solution was retained (Table 2). The first component consisted of subscales from IDAS and STAI, the social concerns subscale of the ASI-3, and the emotion control subscale of the ACQ-R. The items on these measures are associated with depressed mood, anxiety, stress, lethargy, fear of negative evaluation from others, and difficulty regulating negative emotions. Accounting for $53.44 \%$ of the variance, we labeled this component as GNA. The second component included subscales from the PASS-20, PCS, and the physical concerns subscale of the ASI-3. This component was characterized by fear of pain, fear that pain will lead to serious or catastrophic outcomes, physiological responses to pain, brooding about pain, pessimism about the ability to deal with pain, pain-related avoidance, and fear of physical sensations that may result in catastrophic outcomes. Accounting for $10.95 \%$ of the variance, we labeled this component as PNA.

Relationships between negative affect and anxious reactivity GNA was positively related to anxiety $\left[r_{(81)}=.22, p<.05\right]$, but not to SCRs $\left[r_{(81)}=-.11, p=.30\right]$ or exploratory behavior $\left[r_{(81)}=-.01\right.$, $p=.90]$ during shock anticipation. PNA was positively related to anxiety $\left[r_{(81)}=.30, p<.01\right]$ and SCRs $\left[r_{(81)}=.22, p<.05\right]$, and negatively to exploratory behavior $\left[r_{(81)}=-.34, p<.01\right]$. The difference between the correlations for PNA and GNA were significant for SCRs $[z=-2.11, p<.05]$ and exploratory behavior $[z=-2.18, p<.05]$, but not anxiety $[z=-.54, p=.59]$. Controlling for group differences, GNA and PNA were unrelated to anxiety $\left[r_{(81)}=.05, p=.68 ; r_{(81)}=.20, p=.06\right]$, while PNA remained significantly related to SCRs and exploratory behavior $\left[r_{(81)}=.23\right.$, $\left.p<.05 ; r_{(81)}=-.29, p<.01\right]$.

Imaging results

Whole-brain analyses of the task's main effects revealed greater activation for Threat compared with Safe condition in the Al, BNST/caudate/thalamus, aMCC, PCC, and the midbrain in the vicinity of PAG among other regions (Table S3, Figure S2). Several regions exhibited greater activation during the safe context, including the vmPFC and anterior hippocampus (Table S3, Figure S2). With both factors included in the model, GNA is related to increased BOLD responses (Threat-Safe) in the left precuneus (IPRE) and right medial frontal gyrus (rMFG), whereas PNA is related to increased responses in the bilateral dAI (Table 3; Fig. 2). Anxious reactivity was unrelated to IPRE [anxiety: $r_{(81)}$ 


\begin{tabular}{|c|c|c|}
\hline Subscale & $\begin{array}{l}\text { General negative } \\
\text { affect }\end{array}$ & $\begin{array}{l}\text { Pain-related negative } \\
\text { affect }\end{array}$ \\
\hline IDAS General Depression & .91 & .33 \\
\hline IDAS Dysphoria & .87 & .35 \\
\hline IDAS Lassitude & .85 & .21 \\
\hline STAI-Y Trait Anxiety & .80 & .44 \\
\hline IDAS Social Anxiety & .80 & .22 \\
\hline IDAS Traumatic Intrusions & .79 & .18 \\
\hline ASI-3 Social Concerns & .76 & .32 \\
\hline IDAS Insomnia & .75 & .30 \\
\hline STAI-Y State Anxiety & .71 & .46 \\
\hline ACQ-R Emotion Control & -.70 & -.56 \\
\hline IDAS Panic & .70 & .21 \\
\hline IDAS Suicidality & .64 & .11 \\
\hline IDAS Appetite Loss & .63 & .08 \\
\hline IDAS III Temper & .63 & .33 \\
\hline ASI-3 Cognitive Concerns & .53 & .51 \\
\hline PASS-20 Fear & .11 & .86 \\
\hline PCS Magnification & .20 & .85 \\
\hline $\begin{array}{l}\text { PASS-20 Physiological } \\
\text { Anxiety }\end{array}$ & .19 & .83 \\
\hline PASS-20 Cognitive Anxiety & .31 & .82 \\
\hline PCS Rumination & .29 & .79 \\
\hline PCS Helplessness & .29 & .76 \\
\hline $\begin{array}{l}\text { PASS-20 Escape/ } \\
\text { Avoidance }\end{array}$ & .15 & .74 \\
\hline ASI-3 Physical Concerns & .31 & .64 \\
\hline ACQ-R Threat Control & -.47 & -.56 \\
\hline ACQ-R Stress Control & -.50 & -.54 \\
\hline IDAS Appetite Gain & .29 & .34 \\
\hline
\end{tabular}

Salient loadings: $\geq .8$ in bold

$A C Q-R$ Anxiety Control Questionnaire-Revised, ASI-3 Anxiety Sensitivity Index-3, IDAS Inventory of Depression and Anxiety Symptoms, PASS-20 Pain Anxiety Symptoms Scale-20, PCS Pain Catastrophizing Scale, STAI-Y StateTrait Anxiety Inventory-Form $Y$

$=-.08, p=.50 ;$ SCRs: $r_{(81)}=.05, p=.64$; exploratory behavior: $r_{(81)}$ $=-.01, p=.95$ ] and rMFG [anxiety: $r_{(81)}=.21, p=.06$; SCRs: $r_{(81)}$ $=.12, p=.29$; exploratory behavior: $r_{(81)}=.11, p=.33$ ] activity. Conversely, greater anxiety and SCRs were related to increased activity in both left $\mathrm{dAl}\left[r_{(81)}=.39, p<.001 ; r_{(81)}=.55, p<.001\right]$ and right $\mathrm{dAl}\left[r_{(81)}=.34, p<.01 ; r_{(81)}=.51, p<.001\right]$, while reduced exploratory behavior was related to increases in the left dAI activity alone $\left[r_{(81)}=-.21, p=.05\right.$; right dAl activity: $r_{(81)}=-.17$, $p=.13]$. When controlling for group effects, the relationship between anxiety and bilateral dAl [left: $r_{(81)}=.35, p<.005$; right: $\left.r_{(81)}=.28, p<.05\right]$, and SCRs and bilateral dAI [left: $r_{(81)}=.55$, $p<.001$; right: $\left.r_{(81)}=.52, p<.001\right]$ remained significant, while exploratory behavior was unrelated to bilateral dAl activity [left: $r_{(81)}=-.17, p=.11$; right: $r_{(81)}=-.12, p=.28$ ].

Mediation analysis results

Mediation analyses (Fig. 3, Table S4) indicated that the relationship between PNA and anxiety, and PNA and SCRs was partly driven by the effect of activity in the left and right dAI ROls. There was no evidence that PNA influenced anxiety and SCR independent of their effect on the left and right dAl. Mediation analyses yielded no support for an indirect effect of PNA on exploratory behavior through bilateral dAl activity (Table S5), while the direct effect of PNA was significant for bilateral dAl.

\section{DISCUSSION}

In this study, we first derived latent variables of negative affect using a PCA. Next, we examined the relationship between these variables and neural, physiological, and behavioral responses to anticipate the unpredictable shock in a transdiagnostic sample of patients with depressive and anxiety spectrum disorders and healthy individuals. Several findings emerged. PCA of negative valence measures resulted in the extraction of two components, GNA and PNA. Patients had significantly greater GNA and PNA than healthy individuals. During shock anticipation, higher PNA was associated with increases in anxiety and SCRs, as well as reduced exploratory behavior, while higher GNA was only associated with increases in anxiety. PNA was associated with elevated activation in the bilateral dAl, while GNA was related to elevated activation in the left precuneus and right MFG. Finally, bilateral dAI mediated the relationship between PNA and anxiety, and PNA and SCRs.

The PCA results suggested a two-component solution, partly replicating the previous research's three-component solutions (i.e., general distress, fear of pain from injury/insult, and cognitive intrusion of pain) $[17,18]$. Items loading on the GNA component related to depressed mood, anxiety, stress, lethargy, fear of negative evaluation from others, and difficulty regulating negative emotions are similar to the general distress component found previously. Conversely, the PNA component included items related to fear of pain, fear that pain will lead to catastrophic outcomes, fear of physiological responses to pain, brooding about pain, pessimism about the ability to deal with pain, pain-related avoidance, and fear of physical sensations that may result in catastrophic outcomes. This component appears to more closely reflect the cognitive intrusion of pain component, with the exception of fear of physical sensations (ASI-3 physical concerns), which loaded on the fear of pain from injury/insult component in previous research. We did not use the Fear of Pain Questionnaire, whose subscales most strongly loaded on the fear of pain from injury/insult component in these past studies. We also included individuals with depression and anxiety disorders, and together, these factors may have contributed to a two-component rather than three-component solution. Nevertheless, ours and previous research both suggest that PNA is distinct from GNA, and our data show that both are indicative of affective dysfunction. However, while GNA was related to anxiety only, PNA also predicted increased SCRs and decreased exploratory behavior during shock anticipation. Correlations between task-related anxious reactivity and PNA were significantly greater than those with GNA. Therefore, PNA may be a more robust predictor of arousal and behavioral responses when anticipating events that may result in physical discomfort or pain. Conversely, GNA may be a characteristic of pervasive anxious states.

Neuroimaging data showed that elevated GNA was related to increased activity in the precuneus and MFG during shock anticipation. Precuneus plays a critical role in the default mode network and is active during self-referential processes, spatially guided behavior, mental imagery, and episodic/autobiographical memory retrieval $[30,31]$. Precuneus also has been previously implicated in the anticipation of aversive stimuli, including pain $[20,32-34]$. On the other hand, MFG has been found to subserve executive function processes, such as reorienting of attention [35], working memory [36], and contingency awareness during conditioning [37]. It has been also implicated in pain processing [32] and anticipation of aversive stimuli [38], and shows relative increases in activity during anticipation of aversive stimuli in patients $[33,39]$. While both were related to GNA during shock anticipation, neither region was associated with measures of task- 


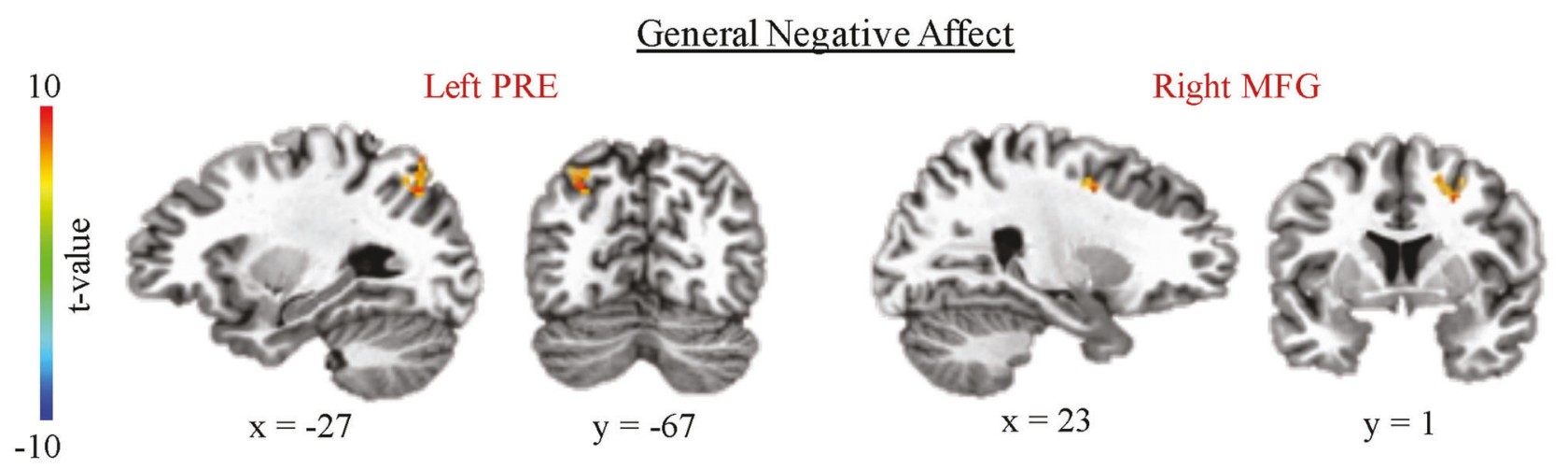

Pain-related Negative Affect

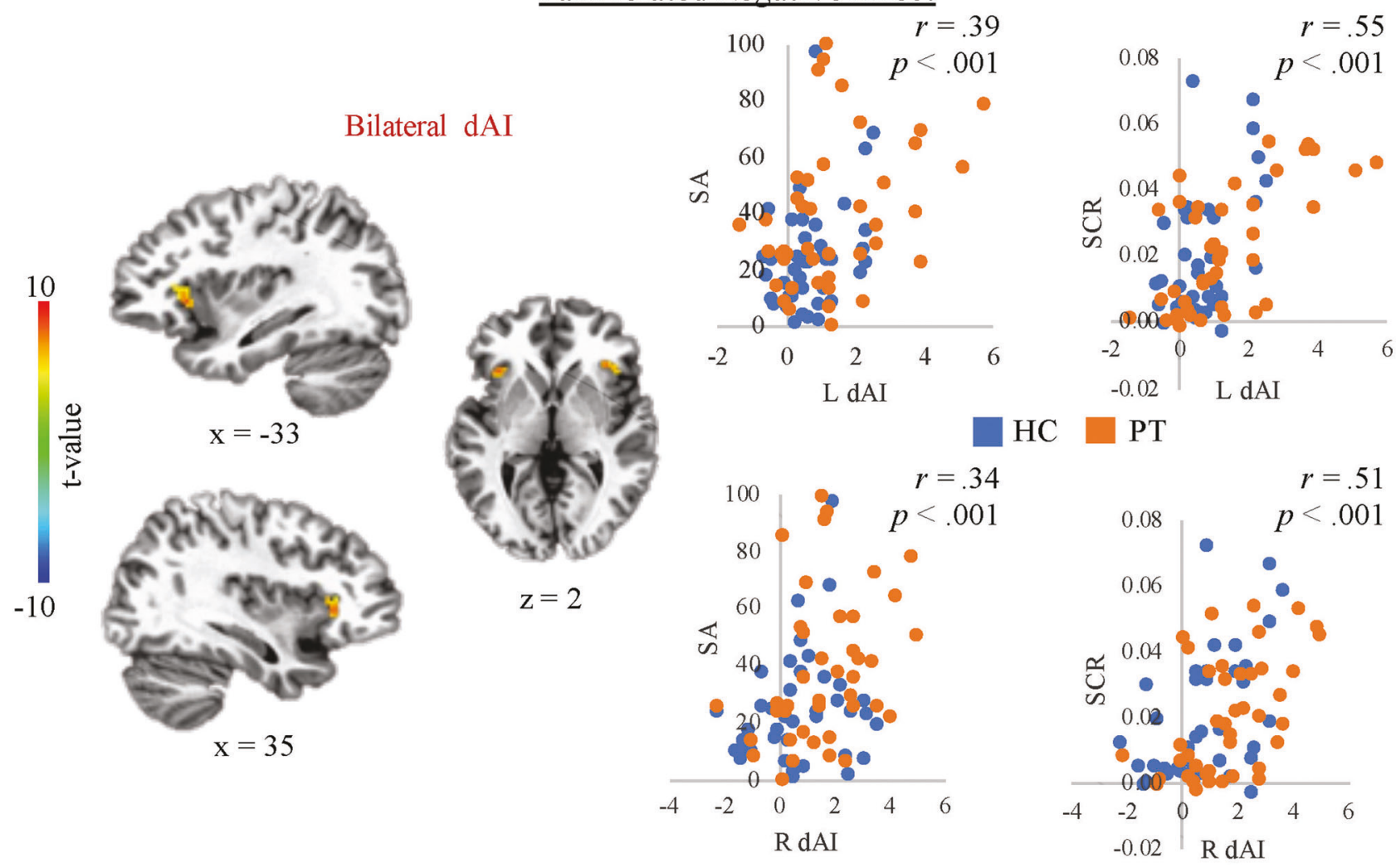

Fig. 2 During anticipation of unpredictable, painful shock, general negative affect (GNA) was related to increased hemodynamic activity in the left precuneus (IPRE) and right middle frontal gyrus (rMFG), while pain-related negative affect was related to increased hemodynamic activity in the bilateral dorsal anterior insula (dAl). Greater hemodynamic responses to anticipation of unpredictable shock in the left and right dAl were related to greater SA and SCRs across all participants. Left is left

related anxious reactivity (i.e., subjective ratings, SCRs, and exploratory behavior). Thus, we posit that these findings reflect a role of precuneus and MFG in attention, self-focus, mental imagery, and/or memory retrieval of internal states under anticipation of physical discomfort or pain.

Conversely, PNA was related to elevated activity in bilateral dAI during shock anticipation. Left and right dAl activity was further associated with elevations in anxiety and SCRs, while the left dAI was also related to a reduction in exploratory behavior. Finally, independent of group effects, left and right dAl mediated the relationship between PNA and anxiety and SCRs. The cortical and subcortical connections of the insular cortex allow for a representation and integration of sensory-discriminative, interoceptive, cognitive, and affective information to create subjective feeling states [40, 41]. Structurally distinct from its middle and posterior regions primarily involved in somatosensory aspects of pain, anterior insula is thought to play a role in cognitive-affective and interoceptive processes by integrating into an emotional experience past knowledge with stimulus salience, its context, and expected impact and outcomes [40, 42]. Further distinctions can be made between the function of the ventral and the dAl. While the ventral insula is involved in processing of emotion, the dAI subserves cognitive functions, such as sustained attending to stimuli, goal monitoring, and modulation of arousal [43]. Therefore, $d A$ i is well positioned to engage in detection and evaluation of noxious stimuli and direct appropriate responses. Indeed, anterior insula responses have been observed during sustained anticipation of aversive images and sounds [44, 45], as well as thermal [46] and electric painful stimuli [47]. Previous research has also reported that depression and anxiety relate to increased anterior insula activity during pain anticipation $[33,39]$. Our results support these findings and further suggest that anterior insula 

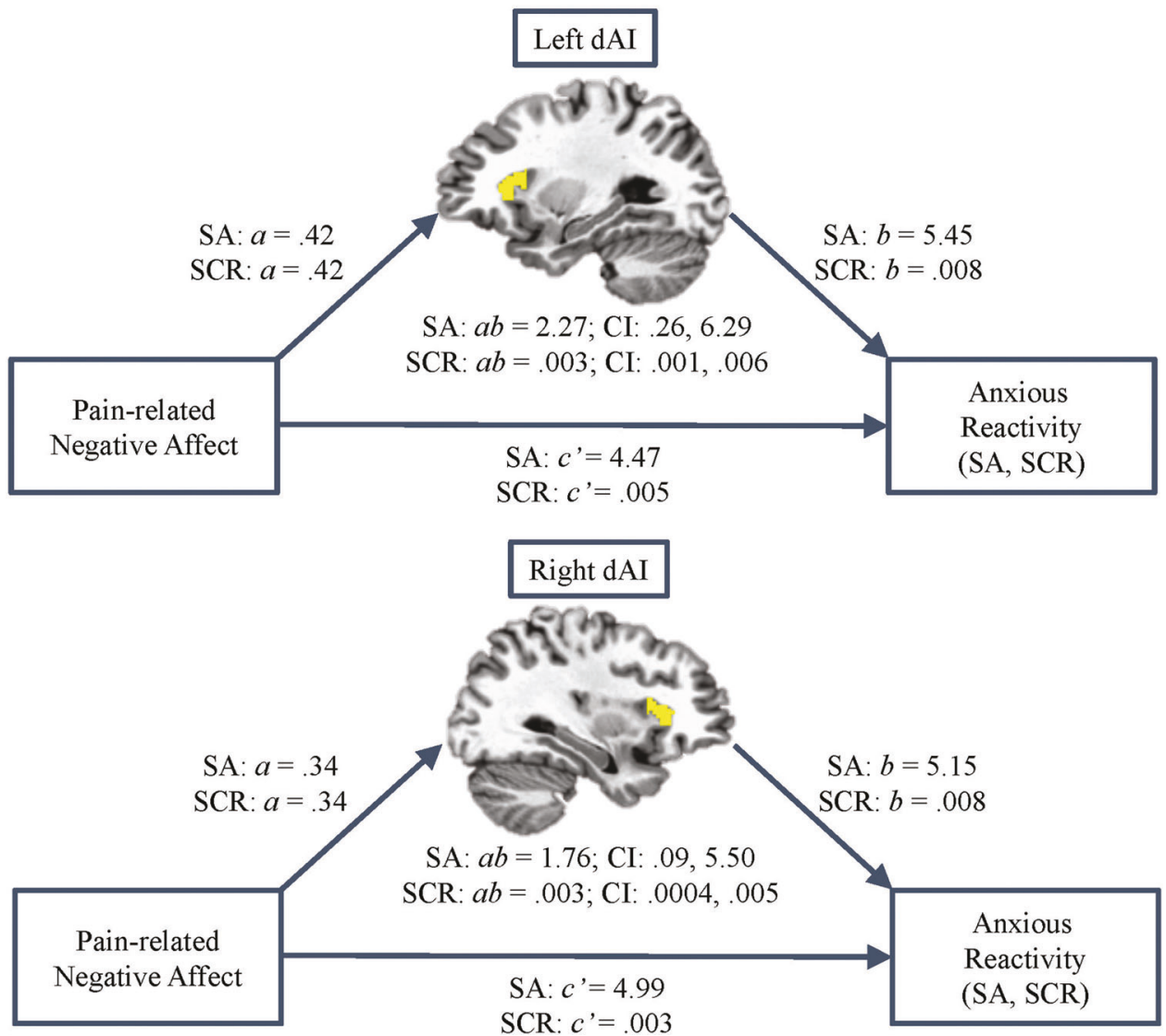

Fig. 3 The pathways from pain-related negative affect (PNA) to the left and right dorsal anterior insula (dAl; path a), and then from dAl to subjective anxiety (SA) and skin conductance responses (SCRs; path b) represent indirect effects of PNA on anxious reactivity through dAI activity, respectively (quantified as the product of paths $\mathbf{a}$ and $\mathbf{b}$ ). Pathway $\mathbf{c}$ represents the direct effect of PNA on anxious reactivity. Model coefficients are reported in an unstandardized form, thus they map directly onto the measurement scales used. A 95\% confidence interval (CI) for the indirect effect $(\mathrm{ab})$ does not contain and is entirely above zero providing evidence that dAl serves as a mediator of the effect of painrelated negative affect on anxious reactivity

Table 3. Regions exhibiting activation differences during anticipation of unpredictable, painful shock (Threat $>$ Safe) that were associated with general (GNA) or pain-related negative affect (PNA)

\begin{tabular}{lllllll}
\hline $\begin{array}{l}\text { Component and hemisphere/ } \\
\text { location }\end{array}$ & $\begin{array}{l}\text { Peak } \\
\text { coordinates }\end{array}$ & & $t_{81}$ & $\begin{array}{l}\text { No. of } \\
\text { voxels }\end{array}$ \\
\cline { 2 - 3 } & $x$ & $y$ & $z$ & & \\
\hline GNA & & & & & & \\
L precuneus & -27 & -67 & 38 & 4.19 & 73 \\
R middle frontal gyrus (MFG) & 23 & 1 & 40 & 4.21 & 48 \\
PNA & & & & & & \\
R dorsal anterior insula & 35 & 23 & 2 & 4.38 & 87 \\
L dorsal anterior insula & -33 & 20 & 2 & 4.52 & 63 \\
\hline
\end{tabular}

${ }^{a}$ All coordinates reported according to stereotaxic array of Talairach and Tournoux [29]. The $x, y, z$ coordinates indicate the distance in millimeters from the anterior commissure in three dimensions: $x$, right-to-left; $y$, anterior-toposterior; $z$, dorsal-to-ventral, with positive values indicating right, anterior, or dorsal and negative values left, posterior, or ventral, respectively. The number of voxels in each cluster reflects contiguous voxels, in which $p<.005$ after applying appropriate corrections for multiple testing

may be directly involved in the cognitive processing of anticipation of physical discomfort or pain and the subsequent elevations in physiological and affective reactivity often observed in patient populations.
A decrease in exploratory behavior is commonly thought to be associated with anxiety-related responses [48]. Although a reduction in exploratory behavior was related to activity in dAl during shock anticipation, dAl did not mediate the relationship between PNA and exploratory behavior. This suggests that while $\mathrm{dAI}$ contributes to the anxious feeling state, it may act in concert with other regions (e.g., aMCC [21]) to mediate behavioral responses associated with these anxious states. Therefore, it is possible that elevated $\mathrm{dAl}$ and the associated physiological and affective reactivity, as well as connections with other regions or networks may contribute over time to maladaptive coping strategies (e.g., excessive withdrawal, safety behaviors, etc.) when anticipating physical discomfort or pain. This, in turn, may result in a range of negative outcomes (e.g., disrupted safety learning and healthy activity, limited positive emotional experiences, social connections, and occupational opportunities) and serve to maintain symptomatology. Paradigms that more explicitly measure exploratory or approach-avoidance responses during threat may be able to clarify the potential relationship between insula and exploratory behavior.

The presented findings have several clinical implications. First, dysregulated pain processing, characterized by fear and avoidance of physical sensations and pain, may uniquely contribute to etiology of depression and anxiety. Indeed, patients with mood and anxiety spectrum disorders in our sample evidenced elevations on the PNA factor. Therefore, assessment of painrelated cognitions and behaviors may prove beneficial in the conceptualization and treatment of these disorders. Second, 
intervention techniques aimed at increasing interoceptive awareness and reducing experiential avoidance of pain (e.g., mindfulness and exposure to physical sensations/discomfort) may have an attenuating effect on the anterior insula activity during anticipation of potentially painful events (which was significantly elevated among patients with mood and anxiety spectrum disorders relative to healthy individuals), thereby reducing arousal and improving the overall outcomes. Future research is needed to test these relationships, as well as whether such approaches may enhance the outcomes of interventions targeting distorted cognitive processes about pain, its consequences, and distress in general (e.g., cognitive therapy). Longitudinal studies would be able to test the predictive utility of PNA on the development of affective disorders, further establishing a distinction between healthy (i.e., adaptive) levels of pain-related anxiety and the levels that constitute vulnerability for the development of psychopathology.

The following limitations should be considered. First, our data are cross-sectional and therefore preclude definitive conclusions regarding causal influences between PNA, anterior insula, and physiological and affective reactivity. Second, although we included a sufficiently large sample $(n=83)$ for fMRI analyses, it was somewhat smaller as compared to other studies employing PCA. Subjects did not complete an exhaustive list of negative valence measures, perhaps not fully elucidating various negative affect components. Finally, future examinations of the specific effect of PNA on pain modulation are warranted, as the present study was designed to specifically examine the effect of PNA and GNA on anticipation of a painful stimulus.

In summary, our data show that PNA may increase arousal and withdrawal in ways that could contribute to the development and maintenance of psychopathology. Further, the present study provides evidence that anterior insula plays an important role in this relationship. Therefore, patient outcomes may be improved by integrating techniques that may target anterior insula and/or result in attenuation of responses to anticipatory pain or discomfort.

\section{FUNDING}

This research was supported by the Laureate Institute for Brain Research and the William K. Warren Foundation.

\section{ADDITIONAL INFORMATION}

Supplementary Information accompanies this paper at (https://doi.org/10.1038/ s41386-018-0187-5).

Competing interests: The authors declare no competing interests.

Publisher's note: Springer Nature remains neutral with regard to jurisdictional claims in published maps and institutional affiliations.

\section{REFERENCES}

1. Kessler RC, Petukhova M, Sampson NA, Zaslavsky AM, Wittchen HÄ. Twelve , Äêmonth and lifetime prevalence and lifetime morbid risk of anxiety and mood disorders in the United States. Int J Methods Psychiatr Res. 2012;21:169-84.

2. Friedrich M. Depression is the leading cause of disability around the world. JAMA. 2017;317:1517.

3. Brown TA, Barlow DH. A proposal for a dimensional classification system based on the shared features of the DSM-IV anxiety and mood disorders: implications for assessment and treatment. Psychol Assess. 2009:21:256-71.

4. Bogduk HMaN, (ed). Classification of chronic pain. Seattle: IASP Press; 1994.

5. Keefe FJ, Lumley M, Anderson T, Lynch T, Carson KL. Pain and emotion: new research directions. J Clin Psychol. 2001;57:587-607.

6. Banks SM, Kerns RD. Explaining high rates of depression in chronic pain: a diathesis-stress framework. Psychol Bull. 1996;119:95.
7. Geisser ME, Roth RS, Theisen ME, Robinson ME, Riley JL III. Negative affect, selfreport of depressive symptoms, and clinical depression: relation to the experience of chronic pain. Clin J Pain. 2000;16:110-20.

8. Asmundson GJ, Katz J. Understanding the co-occurrence of anxiety disorders and chronic pain: state-of-the-art. Depress Anxiety. 2009;26:888-901.

9. Tang J, Gibson SJ. A psychophysical evaluation of the relationship between trait anxiety, pain perception, and induced state anxiety. J Pain. 2005;6:612-9.

10. Terry EL, DelVentura JL, Bartley EJ, Vincent AL, Rhudy JL. Emotional modulation of pain and spinal nociception in persons with major depressive disorder (MDD). PAIN $^{\circledast}$. 2013;154:2759-68.

11. Bair MJ, Robinson RL, Katon W, Kroenke K. Depression and pain comorbidity: a literature review. Arch Intern Med. 2003;163:2433-45.

12. Lilienfeld SO, Turner SM, Jacob RG. Anxiety sensitivity: an examination of theoretical and methodological issues. Adv Behav Res Ther. 1993;15:147-83.

13. Vancleef LM, Vlaeyen JW, Peters ML. Dimensional and componential structure of a hierarchical organization of pain-related anxiety constructs. Psychol Assess. 2009;21:340.

14. Ocañez KL, Kathryn McHugh R, Otto MW. A meta-analytic review of the association between anxiety sensitivity and pain. Depress Anxiety. 2010;27:7 60-7.

15. Carleton RN, Abrams MP, Asmundson GJ, Antony MM, McCabe RE. Pain-related anxiety and anxiety sensitivity across anxiety and depressive disorders. J Anxiety Disord. 2009;23:791-8.

16. Tull MT, Gratz KL. Further examination of the relationship between anxiety sensitivity and depression: the mediating role of experiential avoidance and difficulties engaging in goal-directed behavior when distressed. J Anxiety Disord. 2008;22:199-210.

17. Moore DJ, Eccleston C, Keogh E. Does sex moderate the relationship between anxiety and pain? Psychol Health. 2013;28:746-64.

18. Mounce C, Keogh E, Eccleston C. A principal components analysis of negative affect-related constructs relevant to pain: evidence for a three component structure. J Pain. 2010;11:710-7.

19. Grupe DW, Nitschke JB. Uncertainty and anticipation in anxiety: an integrated neurobiological and psychological perspective. Nat Rev Neurosci. 2013;14:488-501.

20. Palermo S, Benedetti F, Costa T, Amanzio M. Pain anticipation: an activation likelihood estimation meta-analysis of brain imaging studies. Hum Brain Mapp. 2015;36:1648-61.

21. Alvarez RP, Kirlic N, Misaki M, Bodurka J, Rhudy JL, Paulus MP, et al. Increased anterior insula activity in anxious individuals is linked to diminished perceived control. Transl Psychiatry. 2015;5:e591.

22. Roy M, Piche M, Chen J-I, Peretz I, Rainville P. Cerebral and spinal modulation of pain by emotions. Proc Natl Acad Sci USA. 2009;106:20900-5.

23. Fowles DC, Christie MJ, Edelberg R, Grings WW, Lykken DT, Venables PH. Publication recommendations for electrodermal measurements. Psychophysiology. 1981;18:232-9.

24. Bach DR, Flandin G, Friston KJ, Dolan RJ. Time-series analysis for rapid eventrelated skin conductance responses. J Neurosci Methods. 2009;184:224-34.

25. Tabchnick BG, Fidell LS. Using multivariate statistics. Boston: Allyin \& Bacon; 2006.

26. Horn JL. A rationale and test for the number of factors in factor analysis. Psychometrika. 1965;30:179-85.

27. Guadagnoli E, Velicer WF. Relation to sample size to the stability of component patterns. Psychol Bull. 1988;103:265.

28. Hayes AF. Introduction to mediation, moderation, and conditional process analysis: a regression-based approach. New York, NY: The Guilford Press; 2013.

29. Talairach J, Tournoux P. Co-planar stereotaxic atlas of the human brain. New York, NY: Thieme Medical Publishers; 1988.

30. Cavanna AE, Trimble MR. The precuneus: a review of its functional anatomy and behavioural correlates. Brain. 2006;129:564-83.

31. Utevsky AV, Smith DV, Huettel SA. Precuneus is a functional core of the defaultmode network. J Neurosci. 2014;34:932-40.

32. Atlas LY, Lindquist MA, Bolger N, Wager TD. Brain mediators of the effects of noxious heat on pain. PAIN ${ }^{\circledR}$. 2014;155:1632-48.

33. Simmons AN, Flagan TM, Wittmann M, Strigo IA, Matthews SC, Donovan $\mathrm{H}_{\text {, et al }}$ The effects of temporal unpredictability in anticipation of negative events in combat veterans with PTSD. J Affect Disord. 2013;146:426-32.

34. Strigo IA, Simmons AN, Matthews SC, Arthur D, Paulus MP. Association of major depressive disorder with altered functional brain response during anticipation and processing of heat pain. Arch Gen Psychiatry. 2008;65:1275-84.

35. Japee S, Holiday K, Satyshur MD, Mukai I, Ungerleider LG. A role of right middle frontal gyrus in reorienting of attention: a case study. Front Syst Neurosci. 2015;9:23.

36. Hampson M, Driesen NR, Skudlarski P, Gore JC, Constable RT. Brain connectivity related to working memory performance. J Neurosci. 2006;26:13338-43. 
702

37. Carter RM, O'doherty JP, Seymour B, Koch C, Dolan RJ. Contingency awareness in human aversive conditioning involves the middle frontal gyrus. Neuroimage. 2006;29:1007-12.

38. Brooks JC, Nurmikko TJ, Bimson WE, Singh KD, Roberts N. fMRI of thermal pain: effects of stimulus laterality and attention. Neuroimage. 2002; 15:293-301.

39. Strigo I, Matthews S, Simmons A. Decreased frontal regulation during pain anticipation in unmedicated subjects with major depressive disorder. Transl Psychiatry. 2013;3:e239.

40. Kurth F, Zilles K, Fox PT, Laird AR, Eickhoff SB. A link between the systems: functional differentiation and integration within the human insula revealed by meta-analysis. Brain Struct Funct. 2010;214:519-34.

41. Paulus MP, Stein MB. An insular view of anxiety. Biol Psychiatry. 2006;60:383-7.

42. Craig AD, Craig A (2009). How do you feel-now? The anterior insula and human awareness. Nat Rev Neurosci 2009;10:59-70.
43. Chang $\amalg$, Yarkoni T, Khaw MW, Sanfey AG. Decoding the role of the insula in human cognition: functional parcellation and large-scale reverse inference. Cereb Cortex. 2012;23:739-49.

44. Carlson JM, Greenberg T, Rubin D, Mujica-Parodi LR. Feeling anxious: anticipatory amygdalo-insular response predicts the feeling of anxious anticipation. Soc Cogn Affect Neurosci 2010. nsq017 6:74-81.

45. Simmons A, Matthews SC, Stein MB, Paulus MP. Anticipation of emotionally aversive visual stimuli activates right insula. Neuroreport. 2004;15:2261-5.

46. Wiech K, Lin C-s, Brodersen KH, Bingel U, Ploner M, Tracey I. Anterior insula integrates information about salience into perceptual decisions about pain. J Neurosci. 2010;30:16324-31.

47. Chua $\mathrm{P}$, Krams $\mathrm{M}$, Toni $\mathrm{I}$, Passingham R, Dolan R. A functional anatomy of anticipatory anxiety. Neuroimage. 1999;9:563-71.

48. Young J, Minassian A, Paulus MP, Geyer MA, Perry W. A reverse-translational approach to bipolar disorder: rodent and human studies in the Behavioral Pattern Monitor. Neurosci Biobehav Rev. 2007;31:882-96. 